\title{
Automated Angiographic Labeling Pipeline
}

\author{
Jacob Cantrell ${ }^{1}$, Kolten Kersey ${ }^{1}$, Anush Motaganahalli ${ }^{2}$, Amy $\mathrm{Li}^{2}$, Hunter Maxwell ${ }^{2}$, Shantanu \\ Dev $^{2}$, Andrew A. Gonzalez ${ }^{1,2}$ \\ ${ }^{1}$ Indiana University School of Medicine; ${ }^{2}$ Center for Health Service Research, Regenstrief \\ Institute, Inc.
}

\section{Background \& Hypothesis:}

Treatment decisions for medical management, endovascular therapy, open surgery, and hybrid approaches for peripheral artery disease (PAD) are largely driven by imaging. While catheterdirected angiography remains the gold-standard for endoluminal vessel analysis, currently, there is not widespread clinical use of machine learning to provide automated segmentation. This project aims to develop an active learning pipeline to automate the labeling of vascular structures in angiographic images.

\section{Methods:}

We queried the picture archiving and communication system (PACS) database for Indiana University Health and Eskenazi Health to identify studies with catheter-directed angiograms of the extremities. From this dataset we randomly selected an initial convenience sample of 50 angiograms to manually label using the $3 D$ Slicer software. We compared three workflow approaches for labeling this training data - (1) human-only single-pass labelling whereby one person labels each image; (2) human-only multi-pass labelling whereby three humans label a vessel with increasing precision; (3) "human-in-the-middle" approach using NVIDIA's AlAssisted Annotation client whereby the image is auto-segmented and then manually checked for accuracy.

\section{Results:}

We are currently evaluating speed and accuracy for each of these approaches. However, our preliminary data suggests that human-only multi-pass labeling is most efficientappreac We will be validating the following three-step process. First, thresholding tool was used to leverage differences in contrast gradations to approximate the location of vascular structure. Second, the eraser tool was utilized to refine the vessel boundaries. Finally, major blood vessels contributing to axial flow to the foot were manually labeled. These labeled angiograms will be used to develop an active learning algorithm to automate future labeling of the remaining dataset.

\section{Conclusion:}

A machine learning approach to interpreting lower extremity images can dramatically improve the efficiency of triaging patients with PAD. Further work is underway to develop and implement this program clinically. 